would fit better in the second. The point is that foundations deals with general issues of how to conceive and study the mind, whereas domains deals with substantive areas of this study, and language is one such area. A reader not already familiar with cognitive science would be better off reading the latter section first to get a grasp of the research problems, before grappling with the more abstract ways of considering them.

Cognitive science is a meeting place of ideas from computer scientists, psychologists, linguists, philosophers, engineers, neuroscientists, and others interested in the design of intelligent machines, particularly of the machines instantiated by the human brain. In seeking to understand how a person performs a given task, we can start by trying to discover the class of mechanisms that can perform it, or by trying to infer the actual mechanism from performance data. The first tactic lends itself to the style of the computer scientist and engineer, the second tactic to that of the traditional experimental psychologist. The best of recent cognitive science combines these styles, constructing a continuous interaction between theory and data. But though it is not discussed, it becomes evident from reading the book that until recently the theoretical disciplines have made much of the running, producing a cornucopia of models that have left the psychologist flat footed trying to find experimental data to test them. The traditional methods of experimental psychology were simply too narrowly conceived to adapt to the newer ideas. Thus, some of the experimentally based chapters do not quite rise to the occasion, being too myopically concerned with secondary issues.

There is a penalty for this rather one-sided input to the science. Philosophers, computer scientists and linguists have tended to take a cartesian rationalist view of mind, emphasizing its ability to interpret its sensory input and use logical processes to reason and make decisions about an external world. This view follows fairly naturally from the computer metaphor. It is in marked contrast to the earlier cybernetic view of the organism as a control system regulating its world through feedback. The emphasis then was on the organism learning about its world by interacting with and manipulating it. This view also gave prominence to the fact that people in the real world are often confronted with illdefined tasks and problems, and are often required to perform in a timescale that does not allow a rational reflection of options. The range of ill-defined tasks includes such activities as social interaction, artistic creation and scientific research. As soon as we consider these it becomes obvious that we need a theory of improvisation and innovation. This is likely to take a form that extends our current ideas on heuristics, shifting our focus from the search for rules and algorithms.

The signs of a return to the cybernetic view are already apparent in the recent robotics research, where the emphasis is on sen- sory-motor action. A central assumption of the research is that brains form models and representations of their world as bases for action. This is a more dynamic conception than one which regards mental models as bases for interpreting the world. Foundations of Cognitive Science only occasionally picks up this newer trend. Its two chapters on action, postponed to near the end, provide excellent surveys of motor-control theory, but do not tackle the larger issues of mental representation. Hence they fail to counterbalance the cartesian bias of the chapters on, for instance, model-theoretic semantics and mental models. Thus, in a sense the book reflects a phase in the history of cognitive science that may be already passing. But we should be grateful for having it described so expertly.

L. H. Shaffer is in the Department of Psychology, University of Exeter, Exeter EX4 4QG, UK.

\section{Sacred cows}

\section{Bailyn}

Theory of Neutron Star Magnetospheres. By F. Curtis Michel. Chicago University Press: 1991. Pp.517. Hbk \$79.95, £63.95; pbk \$34.95, £27.95.

IT is commonplace in some circles to decry the impersonality of scientific writing. The abstract, dry and totally objective tone of most scientific work, it is claimed, contributes mightily to the double misapprehension that science is both infallible and boring. F. Curtis Michel's monograph Theory of Neutron Star Magnetospheres, a work which challenges not only the conventions of scientific writing, but some of the accepted truths of contemporary astrophysics, is most assuredly neither.

Magnetized neutron stars are thought to be the source of some of the more bizarre patterns of radiation observed in the sky. Among the phenomena commonly attributed to neutron stars are radio pulsars, $\mathrm{X}$-ray bursters, and (by most astronomers) gamma-ray bursters. The surprising observational data on all of these objects has kept theorists off balance for over two decades. Recent discoveries reported in these pages include eclipsing pulsars, globular cluster pulsars, and possible ultra-soft X-ray sources; with the continued improvement in observational capacities at all wavelengths this flood of unexpected objects is likely to continue unabated. Needless to say, theorists have not been shy of offering explanations for all of these phenomena, and in many cases a consensus seems to have been reached. But the foundations on which these theoretical edifices are constructed are often surprisingly weak.

In Theory of Neutron Star Magnetospheres, Michel has taken it upon himself to demonstrate these flaws. Virtually every assumption, hidden or otherwise, invoked in the study of radio pulsars is dissected, and in many cases rejected. I found myself alternatingly delighted at Michel's intellectual courage, and infuriated at his refusal to see reason, depending upon which sacred cow he was engaged in goring. Most researchers in these fields will experience both of these reactions in studying this book, probably in different places from me and from each other. Michel is a clever debater, and has a deep understanding of the rather complex physics underlying pulsar magnetospheres, and I found that the effort of trying to refute some of his seemingly implausible statements deepened my understanding of the theories, and in some cases changed my initially sceptical attitude. Arguing with this book is not a trivial exercise, but it is one which anybody who presumes to write research papers on pulsars should be required to undertake.

But the highly individualistic and argumentative tone of the book has some drawbacks. Chief among these is that it makes the book unsuitable for beginning graduate students, or for researchers in related fields who wish to acquire an overview of current work on neutron stars. This is unfortunate, because the field is advancing rapidly and there is currently a need for a book-length review. But the choice of topics is too idiosyncratic to fulfil this need: a work that dwells for dozens of pages on the speculation that Jupiter should be considered a radio pulsar, but devotes only a single paragraph to the considerable observational and theoretical work devoted to quasiperiodic oscillations in $\mathrm{X}$-ray binaries, which are far more relevant to neutron-star magnetospheres, is hardly a place to begin one's study of the field. To take another example, the many pages devoted to skewering the hypothesis of magnetic-field decay in pulsars would seem pointless to anyone not already steeped in the standard arguments in its favour. The organizational structure is also weak, as Michel leaps between basic physics, phenomenology and observational data, often referring the reader to a crucial figure or argument hundreds of pages forwards in the book. And there are enough of the usual typographical errors and minor misstatements to annoy pedants and trap the unwary.

Despite these quibbles, one must be grateful to Michel for allowing us such unusually close access to his opinions and prejudices. If more scientists were as forthcoming, many fewer mistakes would be repeated and perpetuated. So I hope we will see more such works in the future, in astrophysics and in science generally, despite the treacherous terrain they would present for those not previously acquainted with their subject matter.

C. Bailyn is in the Department of Astronomy, Yale University, PO Box 6666, New Haven Connecticutt 06511, USA 